PROCEEDINGS OF THE

AMERICAN MATHEMATICAL SOCIETY

Volume 137, Number 8, August 2009, Pages 2645-2653

S 0002-9939(09)09892-X

Article electronically published on March 17, 2009

\title{
CONTINUITY OF EXTREMAL ELEMENTS IN UNIFORMLY CONVEX SPACES
}

\author{
TIMOTHY FERGUSON
}

(Communicated by Mario Bonk)

\begin{abstract}
In this paper, we study the problem of finding the extremal element for a linear functional over a uniformly convex Banach space. We show that a unique extremal element exists and depends continuously on the linear functional, and vice versa. Using this, we simplify and clarify Ryabykh's proof that for any linear functional on a uniformly convex Bergman space with kernel in a certain Hardy space, the extremal function belongs to the corresponding Hardy space.
\end{abstract}

Our purpose is to study extremal problems over uniformly convex Banach spaces. For a given linear functional $\phi$, what elements $x$ of the space with norm $\|x\|=1$ maximize $\operatorname{Re} \phi(x)$ ? Because of the uniform convexity, this problem will always have a unique solution, which is called the extremal element. In this paper, we show that the extremal element depends continuously on the functional $\phi$, and it can be approximated by the solutions of the same extremal problem over subspaces of the original Banach space.

Moreover, we show that an extremal element can arise from at most one linear functional of unit norm, and that the functional depends continuously on the extremal element. Using these results, we give a streamlined proof of a theorem of Ryabykh, which says that for a functional defined on the Bergman space $A^{p}$, with kernel in the Hardy space $H^{q}$, the extremal element is in $H^{p}$, where $1<p<\infty$ and $\frac{1}{p}+\frac{1}{q}=1$.

\section{UNIFORM CONVEXITY AND EXTREMAL PROBLEMS}

Let $X$ be a complex Banach space and let $X^{*}$ be its dual space. For a given linear functional $\phi \in X^{*}$ with $\phi \neq 0$, we are interested in all elements $x \in X$ with norm $\|x\|=1$ such that

$$
\operatorname{Re} \phi(x)=\sup _{\|y\|=1} \operatorname{Re} \phi(y)=\|\phi\| .
$$

Such a problem is referred to as an extremal problem, and $x$ is an extremal element.

This problem has been studied extensively for certain Banach spaces. For example, for the Hardy spaces $H^{p}$ where $1 \leq p \leq \infty$, the problem was investigated independently around 1950 by S. Ya. Khavinson, and by Rogosinski and Shapiro. (See Duren [3, Chapter 8.) The problem for the Bergman spaces $A^{p}$ for $1 \leq p<\infty$

Received by the editors September 9, 2008.

2000 Mathematics Subject Classification. Primary 30H05; Secondary 46 B99.

(C)2009 Timothy Ferguson 
has been studied as well, for example by Vukotić [10 and Khavinson and Stessin 6]. We will define the Hardy and Bergman spaces in Section 2 ,

A closely related problem is that of finding $x \in X$ such that

$$
\phi(x)=1 \quad \text { and } \quad\|x\|=\inf _{\phi(y)=1}\|y\| .
$$

If $x$ solves the problem (1.1), then $\frac{x}{\phi(x)}$ solves the problem (1.2), and if $x$ solves (1.2), then $\frac{x}{\|x\|}$ solves (1.1). To standardize notation, we will often denote solutions to (1.1) by $x^{\star}$ and solutions to (1.2) by $x^{\diamond}$.

The problem (1.1) need not have a solution, and if it does, the solution need not be unique. However, if the Banach space $X$ is uniformly convex, there will always be a unique solution.

Definition 1.1. A Banach space $X$ is said to be uniformly convex if for each $\varepsilon>0$, there is a $\delta>0$ such that for all $x, y \in X$ with $\|x\|=\|y\|=1$,

$$
\left\|\frac{1}{2}(x+y)\right\|>1-\delta \quad \text { implies } \quad\|x-y\|<\varepsilon .
$$

An equivalent statement is that if $\left\{x_{n}\right\}$ and $\left\{y_{n}\right\}$ are sequences in $X$ such that $\left\|x_{n}\right\|=\left\|y_{n}\right\|=1$ and $\left\|x_{n}+y_{n}\right\| \rightarrow 2$, then $\left\|x_{n}-y_{n}\right\| \rightarrow 0$. Uniform convexity was introduced by Clarkson [2], who proved that the $L^{p}$ spaces are uniformly convex for $1<p<\infty$.

Proposition 1.2. Let $X$ be a uniformly convex Banach space, and let $\left\{x_{n}\right\}$ and $\left\{y_{n}\right\}$ be sequences in $X$. If for some $d>0,\left\|x_{n}\right\| \rightarrow d$ and $\left\|y_{n}\right\| \rightarrow d$ as $n \rightarrow \infty$, and $\left\|x_{n}+y_{n}\right\| \rightarrow 2 d$, then $\left\|x_{n}-y_{n}\right\| \rightarrow 0$.

Proof. We have that

$$
\begin{aligned}
2 & \geq\left\|\frac{x_{n}}{\left\|x_{n}\right\|}+\frac{y_{n}}{\left\|y_{n}\right\|}\right\|=\frac{1}{\left\|x_{n}\right\|}\left\|x_{n}+\frac{\left\|x_{n}\right\|}{\left\|y_{n}\right\|} y_{n}\right\| \\
& \geq \frac{1}{\left\|x_{n}\right\|}\left\|x_{n}+y_{n}\right\|-\frac{1}{\left\|x_{n}\right\|}\left\|y_{n}-\frac{\left\|x_{n}\right\|}{\left\|y_{n}\right\|} y_{n}\right\| \rightarrow 2
\end{aligned}
$$

as $n \rightarrow \infty$. Hence by uniform convexity,

$$
\left\|\frac{x_{n}}{\left\|x_{n}\right\|}-\frac{y_{n}}{\left\|y_{n}\right\|}\right\| \rightarrow 0
$$

But then

$$
\begin{aligned}
\left\|x_{n}-y_{n}\right\| & =\left\|x_{n}\right\|\left\|\frac{x_{n}}{\left\|x_{n}\right\|}-\frac{y_{n}}{\left\|x_{n}\right\|}\right\| \\
& \leq\left\|x_{n}\right\|\left\|\frac{x_{n}}{\left\|x_{n}\right\|}-\frac{y_{n}}{\left\|y_{n}\right\|}\right\|+\left\|x_{n}\right\|\left\|y_{n}\left(\frac{1}{\left\|x_{n}\right\|}-\frac{1}{\left\|y_{n}\right\|}\right)\right\| \rightarrow 0 .
\end{aligned}
$$

The following result is basic (see for instance [4, Section 2.2) and gives immediately the existence and uniqueness of extremal elements.

Proposition 1.3. A closed convex subset of a uniformly convex Banach space has exactly one element of smallest norm.

Since the problem (1.2) is one of finding an element of minimal norm over a (closed) subspace of the Banach space, it has a unique solution, and thus we obtain the following theorem. 
Theorem 1.4. If $X$ is a uniformly convex Banach space and $\phi \in X^{*}$ with $\phi \neq 0$, then the problems (1.1) and (1.2) both have a unique solution.

For later reference, we record the relations

$$
\begin{gathered}
x^{\star}=\frac{x^{\diamond}}{\left\|x^{\diamond}\right\|}=\|\phi\| x^{\diamond}, \\
x^{\diamond}=\frac{x^{\star}}{\phi\left(x^{\star}\right)}=\frac{x^{\star}}{\|\phi\|}, \\
\|\phi\|=\phi\left(x^{\star}\right)=\frac{1}{\left\|x^{\diamond}\right\|} .
\end{gathered}
$$

We now state a lemma which will be applied repeatedly.

Lemma 1.5. Let $X$ be a uniformly convex Banach space, let $\left\{x_{n}\right\}$ and $\left\{y_{n}\right\}$ be sequences in $X$, and let $\phi \in X^{*}$, where $\phi \neq 0$. If for some $d \geq 0,\left\|x_{n}\right\| \rightarrow d$ and $\left\|y_{n}\right\| \rightarrow d$ as $n \rightarrow \infty$, and if $\left|\phi\left(x_{n}+y_{n}\right)\right| \rightarrow 2 d\|\phi\|$, then $\left\|x_{n}-y_{n}\right\| \rightarrow 0$.

Proof. Since $\left|\phi\left(x_{n}+y_{n}\right)\right| \leq\|\phi\|\left\|x_{n}+y_{n}\right\|$, we have that

$$
\frac{\left|\phi\left(x_{n}+y_{n}\right)\right|}{\|\phi\|} \leq\left\|x_{n}+y_{n}\right\| \leq\left\|x_{n}\right\|+\left\|y_{n}\right\|
$$

But the left and right sides of this inequality both approach $2 d$, so Proposition 1.2 gives the result.

\section{2. $H^{p}$ AND $A^{p}$ SPACES}

We now recall some basic facts about Hardy and Bergman spaces. For proofs and further information, see [3] and [4. Suppose that $f$ is analytic in the unit disc. For $0<p<\infty$ and $0<r<1$, the integral mean is

$$
M_{p}(f, r)=\left\{\frac{1}{2 \pi} \int_{0}^{2 \pi}\left|f\left(r e^{i \theta}\right)\right|^{p} d \theta\right\}^{1 / p}
$$

If $p=\infty$, we write

$$
M_{\infty}(f, r)=\sup _{\theta}\left|f\left(r e^{i \theta}\right)\right| .
$$

For fixed $f$ and $p$, the integral means are increasing functions of $r$. If $\sup _{r} M_{p}(f, r)<$ $\infty$, we say that $f$ is in the Hardy space $H^{p}$. For any function $f$ in $H^{p}$, the radial limit $f\left(e^{i \theta}\right)=\lim _{r \rightarrow 1^{-}} f\left(r e^{i \theta}\right)$ exists for almost every $\theta$. An $H^{p}$ function is uniquely determined by the values of its limit function on any set of positive measure. $H^{p}$ is a Banach space with norm

$$
\|f\|_{H^{p}}=\sup _{r} M_{p}(f, r)=\left\|f\left(e^{i \theta}\right)\right\|_{L^{p}} .
$$

Because of this and the fact that functions in $H^{p}$ are determined by their boundary values, we can consider $H^{p}$ as a subspace of $L^{p}(\mathbb{T})$, where $\mathbb{T}$ denotes the unit circle. Thus, $H^{p}$ is uniformly convex for $1<p<\infty$ and its dual space is isometrically isomorphic to $L^{q} / H^{q}$, where $1 / p+1 / q=1$, with each equivalence class $[g] \in L^{q} / H^{q}$ representing the functional

$$
\phi(f)=\int_{\mathbb{T}} f(z) g(z) d z
$$


The Bergman space $A^{p}$ for $0<p<\infty$ consists of all functions $f$ analytic in the unit disc $\mathbb{D}$ with

$$
\|f\|_{A^{p}}^{p}=\int_{\mathbb{D}}|f(z)|^{p} d \sigma(z)<\infty
$$

where $\sigma$ is Lebesgue measure divided by $\pi$. Because $A^{p}$ is a closed subspace of $L^{p}(\mathbb{D})$, it is uniformly convex for $1<p<\infty$. For $1<p<\infty$, the dual of $A^{p}$ is isomorphic to $A^{q}$, where $1 / p+1 / q=1$, and the element $g \in A^{q}$ represents the functional defined by $\phi(f)=\int_{\mathbb{D}} f(z) \overline{g(z)} d \sigma(z)$. This isomorphism is not an isometry, but if the functional $\phi$ is represented by the function $g \in A^{q}$, then

$$
\|\phi\| \leq\|g\| \leq C_{p}\|\phi\|,
$$

where $C_{p}$ is a constant depending only on $p$. We remark that $H^{p}$ is contained in $A^{p}$, and in fact $\|f\|_{A^{p}} \leq\|f\|_{H^{p}}$.

The following theorem is essentially known (cf. 9]).

Theorem 2.1. Let $X$ be a (closed) subspace of $A^{p}$ for $1<p<\infty$. Then a function $f \in X$ with $\|f\|=1$ solves the extremal problem (1.1) if and only if

$$
\int_{\mathbb{D}} h|f|^{p-1} \overline{\operatorname{sgn} f} d \sigma=0,
$$

for all $h \in X$ with $\phi(h)=0$. More generally, for all $h \in X$,

$$
\int_{\mathbb{D}} h|f|^{p-1} \overline{\operatorname{sgn} f} d \sigma=\frac{\phi(h)}{\|\phi\|} .
$$

Extremal problems for $H^{p}$ are well understood, due to the successful use of "dual extremal problems" (see [3], Chapter 8), but the method does not work as well for $A^{p}$ (see [10]).

\section{Continuous Dependence of the solution on the functional}

It is important to know whether the extremal element depends continuously on the functional. This turns out to be true for uniformly convex spaces.

Theorem 3.1. Suppose that $X$ is a uniformly convex Banach space and that $\left\{\phi_{n}\right\}$ is a sequence of nonzero functionals in $X^{*}$ such that $\phi_{n} \rightarrow \phi \neq 0$. Let $x_{n}^{\star}$ denote the solution to problem (1.1) for $\phi_{n}$, and let $x^{\star}$ be the solution for $\phi$. Similarly, let $x_{n}^{\diamond}$ denote the solution to problem (1.2) for $\phi_{n}$, and let $x^{\diamond}$ be the solution for $\phi$. Then $x_{n}^{\star} \rightarrow x^{\star}$ and $x_{n}^{\diamond} \rightarrow x^{\diamond}$.

Ryabykh [8] gives a different proof of this statement while establishing another theorem, but our proof is simpler and more direct.

Proof. Note that

$$
\phi\left(x_{n}^{\star}\right)=\phi_{n}\left(x_{n}^{\star}\right)+\left(\phi-\phi_{n}\right)\left(x_{n}^{\star}\right)=\left\|\phi_{n}\right\|+\left(\phi-\phi_{n}\right)\left(x_{n}^{\star}\right) \rightarrow\|\phi\|
$$

so that

$$
\phi\left(x_{n}^{\star}+x^{\star}\right) \rightarrow 2\|\phi\| .
$$

Lemma 1.5 now shows that $x_{n}^{\star} \rightarrow x^{\star}$. It follows that $x_{n}^{\diamond} \rightarrow x^{\diamond}$ since

$$
x_{n}^{\diamond}=\frac{x_{n}^{\star}}{\left\|\phi_{n}\right\|} \rightarrow \frac{x^{\star}}{\|\phi\|}=x^{\diamond} \text {. }
$$


For given $\phi$, a unique $x^{\diamond}$ solves the problem (1.2):

$$
\left\|x^{\diamond}\right\|=\min _{\phi(x)=1}\|x\| .
$$

It is natural to ask whether different functionals can give rise to the same solution of the problem (1.2). (Clearly, any two functionals that are positive real multiples of each other will have the same extremal element solving (1.1).) The following theorem answers this question when $X^{*}$ is uniformly convex.

Theorem 3.2. Let $X$ be a Banach space and let $x \in X$ with $x \neq 0$. If $X^{*}$ is uniformly convex, then there exists a unique $\phi \in X^{*}$ such that $x$ solves the problem (1.2) associated with $\phi$.

Proof. By the Hahn-Banach theorem, there is some $\phi \in X^{*}$ such that $\phi(x)=1$ and $\|\phi\|=\frac{1}{\|x\|}$. But if for some $y \in X, \phi(y)=1$, then $1 \leq\|\phi\|\|y\|$, or $\|y\| \geq\|\phi\|^{-1}=$ $\|x\|$. This says that $x$ solves the problem (1.2) associated with $\phi$. To show that $\phi$ is unique, consider the problem of finding $\psi^{\diamond}$ such that

$$
\left\|\psi^{\diamond}\right\|=\min _{\psi \in X^{*}, \psi(x)=1}\|\psi\| .
$$

We claim that if $x$ solves the problem (1.2) for some $\theta \in X^{*}$, then $\theta$ solves the problem (3.1). In particular, $\phi$ solves the problem (3.1). To see this, note that if $x$ solves (1.2) for $\theta$, then $\theta(x)=1$. If $\theta$ is not a solution of (3.1), then there is a functional $\psi$ such that $\|\psi\|<\|\theta\|$ and $\psi(x)=1$. But this is impossible, since it would imply

$$
1=|\psi(x)| \leq\|\psi\|\|x\|=\frac{\|\psi\|}{\|\theta\|}<1,
$$

where we have used the last relation in (1.3). Since $X^{*}$ is uniformly convex, Theorem 1.4 shows that $\phi$ is the unique solution to (3.1), which proves the theorem.

When $x^{\diamond}$ determines the functional $\phi$ uniquely, it is also natural to ask whether $\phi$ depends continuously on $x^{\diamond}$. The following theorem answers this question when $X^{*}$ is uniformly convex.

Theorem 3.3. (a) Suppose that $X$ is a Banach space whose dual space $X^{*}$ is uniformly convex. If $S$ is a closed subspace of $X$, then for any $x \in S$, there exists a unique $\phi \in S^{*}$ such that $x$ solves the problem (1.2) associated with $\phi$ over $S$.

(b) Moreover, if $x_{n} \in S$ and $x_{n} \rightarrow x$, and $\phi_{n}$ is the unique functional in $S^{*}$ that solves the problem (1.2) for $x_{n}$, then $\phi_{n} \rightarrow \phi$.

Proof. Recall that if $S$ is a closed subspace of $X$, then $S^{*}$ is isometrically isomorphic to $X^{*} / S^{\perp}$, where $S^{\perp}$ is the annihilator of $S$ in $X^{*}$. In [7, Section 26, it is shown that the quotient space of a uniformly convex space is uniformly convex, which shows that $S^{*}$ is uniformly convex. From this and Theorem 3.2, part (a) follows.

Since, as shown in the proof of Theorem 3.2, each $\phi_{n}$ is the unique solution to the problem (3.1) with $x_{n}$ in place of $x$, and since $\phi$ is the unique solution of the problem (3.1), Theorem 3.1 implies part (b).

Since $\left(L^{p}\right)^{*}=L^{q}$ is uniformly convex for $1<p<\infty$, this theorem applies to the spaces $A^{p}$ and $H^{p}$ for $1<p<\infty$. 


\section{Approximation by solutions in subspaces AND RYABYKH'S THEOREM}

The preceding results will now be applied to give a streamlined proof of a theorem of Ryabykh [8], as indicated in the introduction. For this purpose it will be helpful to apply the following theorem, which allows an extremal element to be approximated by extremal elements over subspaces.

Theorem 4.1. Suppose that $X$ is a uniformly convex Banach space and let $X_{1}$, $X_{2}, \ldots$ be (closed) subspaces for which $X_{1} \subset X_{2} \subset \cdots \subset X$ and

$$
\overline{\bigcup_{n \in \mathbb{N}} X_{n}}=X
$$

Let $\phi \in X^{*}$, and let

$$
\|\phi\|_{n}=\sup _{x \in X_{n},\|x\|=1}|\phi(x)| .
$$

Let $x_{n}^{\star}$ denote the solution to the problem (1.1) when restricted to the subspace $X_{n}$, and let $x_{n}^{\diamond}$ denote the solution to the problem (1.2) when restricted to $X_{n}$. Then $\|\phi\|_{n} \rightarrow\|\phi\|, x_{n}^{\star} \rightarrow x^{\star}$, and $x_{n}^{\diamond} \rightarrow x^{\diamond}$ as $n \rightarrow \infty$.

Here, $x^{\star}$ denotes the solution to (1.1) over $X$, and $x^{\diamond}$ denotes the solution to (1.2) over $X$.

Proof. First of all, we know that each $x_{n}^{\star}$ and $x_{n}^{\diamond}$ is uniquely determined since a closed subspace of a uniformly convex space is uniformly convex. Let $\varepsilon>0$ be given. Since $\bigcup_{n \in N} X_{n}$ is dense in $X$, we may choose an $n$ such that $\left\|x^{\star}-y\right\|<\varepsilon$ for some $y \in X_{n}$. Thus

$$
\begin{aligned}
|\phi(y)| & =\left|\phi\left(x^{\star}\right)-\phi\left(x^{\star}-y\right)\right| \geq\left|\phi\left(x^{\star}\right)\right|-\left|\phi\left(x^{\star}-y\right)\right|=\|\phi\|-\left|\phi\left(x^{\star}-y\right)\right| \\
& \geq\|\phi\|-\|\phi\|\left\|x^{\star}-y\right\| \geq\|\phi\|(1-\varepsilon) .
\end{aligned}
$$

We also know that $\|y\| \leq 1+\varepsilon$, so

$$
\|\phi\|_{n} \geq \frac{|\phi(y)|}{\|y\|} \geq \frac{(1-\varepsilon)\|\phi\|}{1+\varepsilon}
$$

and thus for all $N \geq n$,

$$
\|\phi\|_{N} \geq \frac{(1-\varepsilon)\|\phi\|}{1+\varepsilon} .
$$

But since $\|\phi\| \geq\|\phi\|_{m}$ for all $m$, this implies that

$$
\|\phi\| \geq \limsup _{m \rightarrow \infty}\|\phi\|_{m} \geq \liminf _{m \rightarrow \infty}\|\phi\|_{m} \geq \frac{(1-\varepsilon)\|\phi\|}{1+\varepsilon} .
$$

Because $\varepsilon$ was arbitrary, this shows that $\|\phi\|_{m} \rightarrow\|\phi\|$.

Now, $\phi\left(x_{n}^{\star}+x^{\star}\right)=\|\phi\|_{n}+\|\phi\| \rightarrow 2\|\phi\|$, so Lemma 1.5 shows that $\left\|x^{\star}-x_{n}^{\star}\right\| \rightarrow 0$. For $x^{\diamond}$, the result now follows since

$$
x_{n}^{\diamond}=\frac{x^{\star}}{\|\phi\|_{n}} \quad \text { and } \quad x^{\diamond}=\frac{x^{\star}}{\|\phi\|} .
$$


With the help of the preceding results, we can now obtain a slightly sharpened version of Ryabykh's theorem. Our proof adapts some of Ryabykh's ideas but is simpler and more concise. We note that Ryabykh's approach successfully applies to other extremal problems as well. For example, it applies to some problems of best approximation of a given function by harmonic or analytic functions (see [5]), and to some nonlinear extremal problems involving nonvanishing functions (see [1]).

Theorem 4.2. Let $1<p<\infty$ and let $1 / p+1 / q=1$. Suppose that $\phi \in\left(A^{p}\right)^{*}$ and $\phi(f)=\int_{\mathbb{D}} f \bar{g} d \sigma$ for some $g \in H^{q}$, where $g \neq 0$. Then the solution to the extremal problem (1.1) (with $X=A^{p}$ ) belongs to $H^{p}$ and satisfies

$$
\left\|f^{\star}\right\|_{H^{p}} \leq\left\{[\max (p-1,1)] \frac{C_{p}\|g\|_{H^{q}}}{\|g\|_{A^{q}}}\right\}^{q / p},
$$

where $C_{p}$ is the constant in (2.1).

Of course, this implies that the solution to the problem (1.2) is in $H^{p}$ as well. Note that the constant $C_{p} \rightarrow \infty$ as $p \rightarrow 1$ or $p \rightarrow \infty$.

Proof. Let

$$
G(z)=\frac{1}{z} \int_{0}^{z} g(\zeta) d \zeta
$$

so that $(z G)^{\prime}=g$.

Now, the continuous form of Minkowski's inequality gives

$$
\begin{aligned}
M_{q}(z G, r) & =\left\{\frac{1}{2 \pi} \int_{0}^{2 \pi}\left|r e^{i \theta} G\left(r e^{i \theta}\right)\right|^{q} d \theta\right\}^{1 / q}=\left\{\frac{1}{2 \pi} \int_{0}^{2 \pi}\left|\int_{0}^{r} g\left(\rho e^{i \theta}\right) e^{i \theta} d \rho\right|^{q} d \theta\right\}^{1 / q} \\
& \leq \int_{0}^{r}\left\{\frac{1}{2 \pi} \int_{0}^{2 \pi}\left|g\left(\rho e^{i \theta}\right)\right|^{q} d \theta\right\}^{1 / q} d \rho \leq \int_{0}^{r} M_{q}(g, \rho) d \rho \leq\|g\|_{H^{q}} .
\end{aligned}
$$

Thus,

$$
\mid G\left\|_{H^{q}} \leq\right\| g \|_{H^{q}} .
$$

We will need the Cauchy-Green theorem, which will allow us to relate the $H^{p}$ and $A^{p}$ spaces. For any $f \in C^{1}(\overline{\mathbb{D}})$, it states that

$$
\frac{1}{2 i} \int_{\mathbb{T}} f(z) d z=\pi \int_{\mathbb{D}} \frac{\partial}{\partial \bar{z}} f(z) d \sigma(z) .
$$

To facilitate calculations involving the Cauchy-Green theorem, we suppose first that $g \in C^{1}(\overline{\mathbb{D}})$. Let $f_{n}^{\star}$ denote the solution to the extremal problem (1.1) over the space of all polynomials of degree $n$ or less, considered as a subspace of $A^{p}$, and let $f^{\star}$ be the solution to the same problem over the space $A^{p}$. Then by the Cauchy-Green theorem,

$$
\begin{aligned}
\left\|f_{n}^{\star}\right\|_{H^{p}}^{p} & =\frac{1}{2 \pi} \int_{0}^{2 \pi}\left|f_{n}^{\star}\left(e^{i \theta}\right)\right|^{p} d \theta=\frac{1}{2 \pi i} \int_{\mathbb{T}}\left|f_{n}^{\star}(z)\right|^{p} \bar{z} d z \\
& =\int_{\mathbb{D}}\left(f_{n}^{\star}+\frac{p}{2} z f_{n}^{\star \prime}\right)\left|f_{n}^{\star}\right|^{p-1} \overline{\operatorname{sgn} f_{n}^{\star}} d \sigma .
\end{aligned}
$$


Because $\left(f_{n}^{\star}+\frac{p}{2} z f_{n}^{\star \prime}\right)$ is a polynomial of degree at most $n$, we can appeal to Theorem 2.1 with $X$ taken to be the subspace of $A^{p}$ consisting of all such polynomials. The theorem shows that

$$
\begin{aligned}
\left\|f_{n}^{\star}\right\|_{H^{p}}^{p} & =\frac{1}{\|\phi\|} \phi\left(f_{n}^{\star}+\frac{p}{2} z f_{n}^{\star \prime}\right)=\frac{1}{\|\phi\|} \int_{\mathbb{D}} \bar{g}\left(f_{n}^{\star}+\frac{p}{2} z f_{n}^{\star \prime}\right) d \sigma \\
& =\frac{1}{\|\phi\|} \int_{\mathbb{D}}\left[\frac{\partial}{\partial \bar{z}}\left(f_{n}^{\star} \overline{z G}\right)+\frac{p}{2}\left(\frac{\partial}{\partial z}\left(z f_{n}^{\star} \bar{g}\right)-\frac{\partial}{\partial \bar{z}}\left(f_{n}^{\star} \overline{z G}\right)\right)\right] d \sigma .
\end{aligned}
$$

Now another application of the Cauchy-Green theorem gives:

$$
\begin{aligned}
\left\|f_{n}^{\star}\right\|_{H^{p}}^{p} & =\frac{1}{2 \pi i\|\phi\|} \int_{\mathbb{T}} \overline{z G} f_{n}^{\star} d z-\frac{p}{4 \pi i\|\phi\|} \int_{\mathbb{T}} z f_{n}^{\star} \bar{g} d \bar{z}-\frac{p}{4 \pi i\|\phi\|} \int_{\mathbb{T}} f_{n}^{\star} \overline{z G} d z \\
& =\frac{1}{2 \pi\|\phi\|} \int_{0}^{2 \pi} f_{n}^{\star}\left[\left(\frac{p}{2}\right) \bar{g}+\left(1-\frac{p}{2}\right) \bar{G}\right] d \theta \\
& \leq \frac{1}{\|\phi\|}\left\|f_{n}^{\star}\right\|_{H^{p}}\left\|\left(\frac{p}{2}\right) g+\left(1-\frac{p}{2}\right) G\right\|_{H^{q}} .
\end{aligned}
$$

Minkowski's inequality now gives

$$
\left\|f_{n}^{\star}\right\|_{H^{p}}^{p} \leq \frac{1}{\|\phi\|}\left\|f_{n}^{\star}\right\|_{H^{p}}\left(\frac{p}{2}\|g\|_{H^{q}}+\left|1-\frac{p}{2}\right|\|G\|_{H^{q}}\right) .
$$

Since $p-1=\frac{p}{q}$, this shows that

$$
\left(\frac{1}{2 \pi} \int_{0}^{2 \pi}\left|f_{n}^{\star}\left(r e^{i \theta}\right)\right|^{p} d \theta\right)^{1 / p} \leq \frac{1}{\|\phi\|^{q / p}}\left(\frac{p}{2}\|g\|_{H^{q}}+\left|1-\frac{p}{2}\right|\|G\|_{H^{q}}\right)^{q / p},
$$

when $0<r<1$. From Theorem 4.1 and the fact that convergence in $A^{p}$ implies uniform convergence on compact subsets of the disc, it follows that

$$
\left(\frac{1}{2 \pi} \int_{0}^{2 \pi}\left|f^{\star}\left(r e^{i \theta}\right)\right|^{p} d \theta\right)^{1 / p} \leq \frac{1}{\|\phi\|^{q / p}}\left(\frac{p}{2}\|g\|_{H^{q}}+\left|1-\frac{p}{2}\right|\|G\|_{H^{q}}\right)^{q / p},
$$

when $0<r<1$. Now we apply (4.2) to infer that

$$
\left\|f^{\star}\right\|_{H^{p}} \leq\left\{\left(\frac{p}{2}+\left|1-\frac{p}{2}\right|\right) \frac{\|g\|_{H^{q}}}{\|\phi\|}\right\}^{q / p} .
$$

Since we know that $\|g\|_{A^{q}} \leq C_{p}\|\phi\|$, and that $p / 2+|1-(p / 2)|=\max (p-1,1)$, we conclude finally that the inequality (4.1) holds under the assumption that $g \in$ $C^{1}(\overline{\mathbb{D}})$.

If $g$ is a general function in $H^{q}$, we can approximate it in the $H^{q}$ norm by a sequence of functions $g_{n} \in C^{1}(\overline{\mathbb{D}})$. (We may even use polynomials, by [3], Theorem 3.3.) Then the corresponding functionals $\phi_{n}$ converge to $\phi$, so by Theorem 3.1, the extremal elements $f_{n}^{\star}$ for $\phi_{n}$ converge to the extremal element $f^{\star}$ for $\phi$ in the $A^{p}$ norm. Since $g_{n} \in C^{1}(\overline{\mathbb{D}})$, we have already found that

$$
\left(\frac{1}{2 \pi} \int_{0}^{2 \pi}\left|f_{n}^{\star}\left(r e^{i \theta}\right)\right|^{p} d \theta\right)^{1 / p} \leq\left\{[\max (p-1,1)] \frac{C_{p}\left\|g_{n}\right\|_{H^{q}}}{\left\|g_{n}\right\|_{A^{q}}}\right\}^{q / p}, \quad 0<r<1 .
$$


But the convergence of $f_{n}^{\star} \rightarrow f^{\star}$ in the $A^{p}$ norm implies that $f_{n}^{\star}(z) \rightarrow f^{\star}(z)$ locally uniformly, so it follows that

$$
\left(\frac{1}{2 \pi} \int_{0}^{2 \pi}\left|f^{\star}\left(r e^{i \theta}\right)\right|^{p} d \theta\right)^{1 / p} \leq\left\{[\max (p-1,1)] \frac{C_{p}\|g\|_{H^{q}}}{\|g\|_{A^{q}}}\right\}^{q / p}, \quad 0<r<1,
$$

which proves (4.1).

\section{REFERENCES}

[1] Dov Aharonov, Catherine Bénéteau, Dmitry Khavinson, and Harold Shapiro, Extremal problems for nonvanishing functions in Bergman spaces, Selected topics in complex analysis, Oper. Theory Adv. Appl., Vol. 158, Birkhäuser, Basel, 2005, pp. 59-86. MR2147588 (2006i:30047)

[2] James A. Clarkson, Uniformly convex spaces, Trans. Amer. Math. Soc. 40 (1936), no. 3, 396-414. MR1501880

[3] Peter Duren, Theory of $H^{p}$ spaces, Pure and Applied Mathematics, Vol. 38, Academic Press, New York, 1970. MR0268655 (42:3552)

[4] Peter Duren and Alexander Schuster, Bergman spaces, Mathematical Surveys and Monographs, Vol. 100, American Mathematical Society, Providence, RI, 2004. MR2033762 (2005c:30053)

[5] Dmitry Khavinson, John E. McCarthy, and Harold S. Shapiro, Best approximation in the mean by analytic and harmonic functions, Indiana Univ. Math. J. 49 (2000), no. 4, 14811513. MR1836538 (2002b:41023)

[6] Dmitry Khavinson and Michael Stessin, Certain linear extremal problems in Bergman spaces of analytic functions, Indiana Univ. Math. J. 46 (1997), no. 3, 933-974. MR.1488342 (99k:30080)

[7] Gottfried Köthe, Topological vector spaces. I, Translated from the German by D. J. H. Garling, Die Grundlehren der Mathematischen Wissenschaften, Band 159, Springer-Verlag New York Inc., New York, 1969. MR.0248498 (40:1750)

[8] V. G. Ryabykh, Extremal problems for summable analytic functions, Sibirsk. Mat. Zh. 27 (1986), no. 3, 212-217, 226 (in Russian). MR853902 (87j:30058)

[9] Harold S. Shapiro, Topics in approximation theory, with appendices by Jan Boman and Torbjörn Hedberg, Lecture Notes in Math., Vol. 187, Springer-Verlag, Berlin, 1971. MR 0437981 (55:10902)

[10] Dragan Vukotić, Linear extremal problems for Bergman spaces, Exposition. Math. 14 (1996), no. 4, 313-352. MR1418027 (97m:46117)

Department of Mathematics, University of Michigan, 2074 East Hall, 530 Church Street, Ann Arbor, Michigan 48109-1043

E-mail address: tjferg@umich.edu 